\title{
Designing a Fuzzy Rule Base System to Head Cluster Election in Wireless Sensor Networks
}

\author{
Maysam Gharghi ${ }^{1 *}$, E. Parvinnia ${ }^{2}$ and R. Khayami ${ }^{3}$ \\ 'Department of Computer, Science and Research Branch, Islamic Azad University, \\ Kerman, Iran; maysam.gharghi@gmail.com \\ 2Department of Computer Engineering, Shiraz Branch, Islamic Azad University, \\ Shiraz, Iran; parvinnia@iaushiraz.ac.ir \\ ${ }^{3}$ Information Technology Department, Shiraz University Of Technology, \\ Shiraz,Iran; khayami@sutech.ac.ir
}

\begin{abstract}
A novel and efficient clustering algorithm in wireless sensor networks is proposed, which can allow the structured representation of a network topology. According to the residual energy, number of the neighbors, and centrality of each node, the algorithm uses fuzzy rule base system to select cluster head. The algorithm not only balances the energy load of each node, but also provides global reliability for the whole networks.
\end{abstract}

Keywords: Clustering Algorithm, Wireless Sensor Network, Fuzzy Rule.

\section{Introduction}

A Wireless sensor network (WSN) is entail of "sensors" which we say nodes - deployed from some sensor nodes to numerous hundreds or even thousands numbers of nodes, where every sensor node is associated by radio link to one or sometimes several other sensors nodes. Each such sensor network node comprises of typically several parts: an electronic circuit to interacting with other sensors nodes and an external energy source (sensors are battery operated), a radio transceiver with an association to an external antenna or may have an embedded internal antenna, a microcontroller and typically an embedded form of energy source or a battery $[1,2]$.

Sensor nodes in WSN are small battery powered devices with limited energy resources, and their batteries cannot be recharged once the sensor nodes are deployed. Therefore, minimizing energy consumption is an important issue in the design of WSN protocols. The energy is also the major consideration in designing the routing of the WSN so that

\footnotetext{
* Corresponding author:

Maysam Gharghi (maysam.gharghi@gmail.com)
}

the lifetime of the networks is maximized. Cluster routing is an effective solution in reducing energy consumption and achieving the network scalability [3]. Recently, many energy efficient clustering routing algorithms have been designed for WSN. LEACH [4] selects cluster heads with some probability.

However, some cluster heads may be very close each other and cannot be uniformly deployed in the networks by probability mechanism, and cluster heads number is not always equal to the pre-established number. PEGASIS [5] organizes sensor nodes into a chain using a greedy algorithm so that each node only communicates with its neighbors. Nevertheless, PEGASIS requires the global knowledge of the network topology and the farther nodes will result in a bigger data delay. HEED [6] introduces a variable known as the cluster radius which defines the transmission power to be used for intracluster broadcast. But it is difficult to realize HEED in practical WSN.

In this paper, an efficient clustering algorithm for wireless sensor networks is presented. A fuzzy inference system 
(FIS) is designed to produce a probability of being head cluster, in the LEACH algorithm. Fuzzy set theory [7] provides a framework to materialize a fuzzy rule base system which contains the selection of fuzzy rules, membership functions, and the reasoning mechanism. Such a system has been applied to many disciplines such as control systems, decision making and pattern recognition [7], and in this paper it is supposed that such a system could overcome the complexity of deciding with considering several parameters. More details are presented in following sections.

\section{Fuzzy Rule Base System}

Among all the techniques developed using fuzzy sets, fuzzy if-then rules are by far the most popular due to their wide range of successful applications. Fuzzy theory provides a framework to materialize a fuzzy rule base system which contains the selection of fuzzy rules, membership functions, and the reasoning mechanism. Such a system has been applied to many disciplines such as control systems, decision making and pattern recognition.

Fuzzy inference system consists of a fuzzification interface, a rule base, a database, a decision-making unit, and finally a defuzzification interface.

The function of each block is as follow [7]:

- A rule base containing a number of fuzzy if-then rules.

- A database which defines the membership functions of the fuzzy sets used in the fuzzy rules.

- A decision-making unit which performs the inference operation on the rules.

- A fuzzification interface which transforms the crisp input in to degrees of match with linguistic values.

- A defuzzification interface which transform the fuzzy results of the interface in to the crisp output.

The crisp input of FIS is converted to fuzzy using fuzzification method. The rule base and the database are jointly referred to as the knowledge base and are formed after the fuzzification. Finally, defuzzification is utilized to convert fuzzy value to the real world value as the output. The steps of fuzzy reasoning (inference operations upon fuzzy IF-THEN rules) performed by FISs are:

1. Comparing the input variables with the membership functions on the antecedent part to obtain the membership values of each linguistic label. (This step is often called fuzzification.)
2. Combining (through a specific $t$-norm operator, usually multiplication or min) the membership values on the premise part to get firing strength (weight) of each rule.

3. Generating the qualified consequents (either fuzzy or crisp) or each rule depending on the firing strength.

4. Aggregating the qualified consequents to produce a crisp output. (This step is called defuzzification.)

The most typical fuzzy inference methods are Mamdani and Sugeno or Takagi-Sugeno-Kung methods. Mamdani type is the most commonly used inference method which is employed in this study as well. Mamdani's advantages can be mentioned as:

- It is intuitive,

- It has widespread acceptance,

- It is well suited the human operator behavior.

Proposed by Mamdani in 1975 as an attempt to control a steam engine and boiler compound by synthesizing a set of linguistic control rules obtained from expert operators, Mamdani’s effort was based on Zadeh's (1973) paper on fuzzy algorithms for complex systems and decision processes.

This method consists of the following linguistic rules:

$R_{i}$ IF $x_{1}$ is $A_{i 1}$ and $\ldots$ and $x_{r}$ is $A_{i r}$ THEN $y$ is $C_{i}$, where $x_{j}$ $(j=1,2, \ldots, r)$ are the input variables, $y$ is the output variable, and $A_{i j}$ and $C_{\mathrm{i}}$ are fuzzy sets for $x_{j}$ and $y$ respectively. Given input of the form: $x_{1}$ is $A_{1}^{\prime}, x_{2}$ is $A_{2}^{\prime}, \ldots, x_{r}$ is $A_{r}^{\prime}$. Where $A_{1}^{\prime}$, $A_{2}^{\prime}, \ldots, A_{r}^{\prime}$ are fuzzy subsets of $U_{1}, U_{2}, \ldots, U_{r}$, the contribution of rule $R_{i}$ to Mamdani model's output is a fuzzy set whose membership function is

$$
\mu_{C_{i}^{\prime}}(y)=\left(a_{i 1} \wedge a_{i 2} \wedge \ldots \wedge a_{i n}\right) \wedge \mu_{C_{i}}(y)
$$

Where $\alpha_{i}$ is the matching degree of rule $R_{i}$, and where $\alpha_{i j}$ is the matching degree between $x_{j}$ and $R_{i}^{\prime}$ s condition on $x_{j}$.

$$
a_{i j}=\sup \left(\mu_{A^{\prime} j}\left(x_{j}\right) \wedge \mu_{A_{i j}}\left(x_{j}\right)\right)
$$

where $\wedge$ denotes the "min" operator.

The final output of the model is the aggregation of outputs from all rules using the max operator;

$$
\mu_{C}(y)=\max \left\{\mu_{C_{1}^{\prime}}(y), \mu_{C_{2}^{\prime}}(y), \ldots, \mu_{C_{L}^{\prime}}(y)\right\}
$$

The output $C$ is a fuzzy set. The fuzzy results generated can not be applied as such, therefore; it is necessary to convert the fuzzy quantities in to crisp quantities for further steps. This can be achieved using defuzzification process. Defuzzification reduces the collection of membership values to a single-valued quantity. 


\section{Proposed Method}

LEACH is a popular current approach for cluster head selection and prepared the basis for many approaches. Algorithm like LEACH uses probabilistic approach to select cluster-head schematically. But this method use only local information to take decision of cluster-head so using only local information has its own limitations. Since each node probabilistically elects whether or not to become cluster-head, there might be cases when two cluster-heads are selected in the closed proximity of each other. In reality considering only one factor like energy, is not suitable to elect the cluster-head properly. This is because other conditions like centrality of nodes corresponding to the entire cluster, gives an amount of the entire dissipation during transmission for all nodes too. The more central the node to a cluster the more is the energy efficiency for other nodes to transmit through that selected node [8].

Here a new cluster-head election scheme "Cluster Head Election using Fuzzy Rule Base System" is designed, which combine various conditions and parameters to determine the chance as system output. To balance the entire nodes energy consumption, designed algorithm constructs clusters at each round similar to LEACH. To get a cluster head election chance, three fuzzy sets and different fuzzy production rules for knowledge representation are considered.

The fuzzy variables that are used in the fuzzy production rules are defined as follows.

1. Residual Energy - the energy remain of each node. The more residual energy the node has, the more data is processed and transmitted, and the longer lifetime of the node is.

2. Number of Neighbors - number of neighbor nodes of each node. The number of neighbor affects in some way for proper cluster head election. It is more reasonable to select a cluster head in a region where the node has more neighbors.

3. Centrality - the sum of distances between the node and the nodes which is within its communication range, which represents how central the node is to the cluster. The more central the node is to a cluster head, the more is the energy efficiency for it to transmit the data through that cluster head. The above fuzzy linguistic variables are input fuzzy variables for the fuzzy production rules, and the output variable is the node's cluster head election chance.
- The fuzzy variable "residual energy" has three fuzzy sets high, medium and low, and its membership function is shown in Figure 1.

- The fuzzy variable "number of neighbors" has three fuzzy sets - many, medium and few. The possible fuzzy quantization of the range [1-10] for the number of neighbors is an experimental result. The membership function is shown in Figure 2.

- The fuzzy variable "centrality" has three fuzzy sets far, medium and close, and its membership function is shown in Figure 3.

- The outcome to represent the node's cluster head election chance has five fuzzy sets -smallest, small, medium, large, and largest, and its membership function is shown in Figure 4.

Since each input variable has 3 linguistic states, the total number of possible fuzzy inference rules is $3 \times 3 \times 3=27$. Some examples of defined rules are as follows:

1. If (residual_energy is low) and (neighbors\# is few) and (centrality is far) then (chance is smallest)

2. If (residual_energy is medium) and (neighbors\# is few) and (centrality is far) then (chance is smallest)

3. If (residual_energy is low) and (neighbors\# is medium) and (centrality is far) then (chance is smallest)

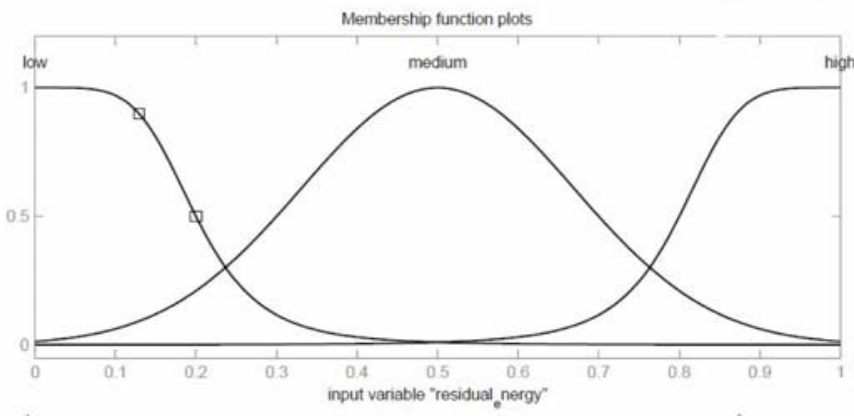

Figure 1. Membership function of first input (residual energy).

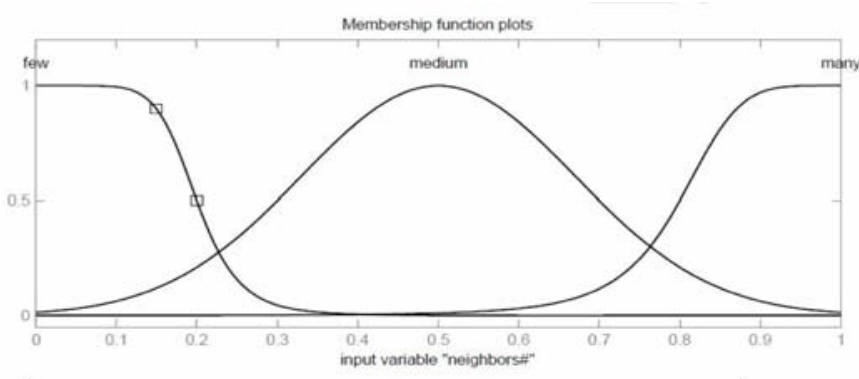

Figure 2. Membership function of second input (number of neighbors). 


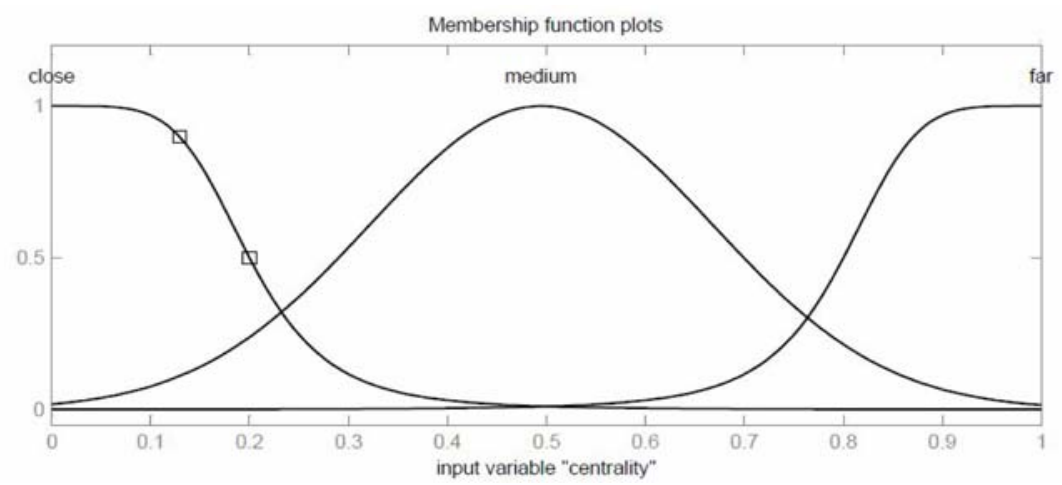

Figure 3. Membership function of third input (centrality).

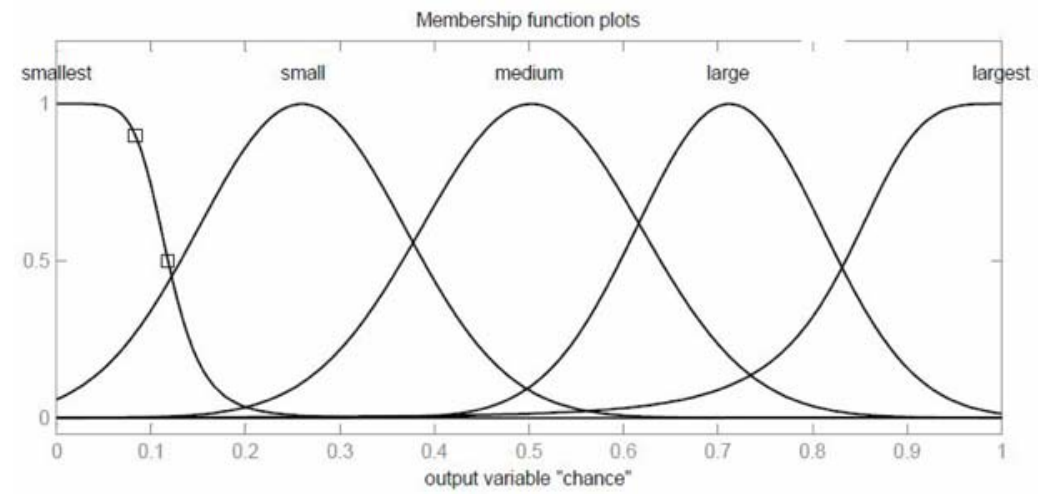

Figure 4. Membership function of output (chance).

4. If (residual_energy is low) and (neighbors\# is few) and (centrality is medium) then (chance is smallest)

5. If (residual_energy is low) and (neighbors\# is medium) and (centrality is medium) then (chance is small)

6. If (residual_energy is medium) and (neighbors\# is medium) and (centrality is far) then (chance is small)

7. If (residual_energy is high) and (neighbors\# is many) and (centrality is close) then (chance is largest)

8. If (residual_energy is high) and (neighbors\# is medium) and (centrality is close) then (chance is large)

9. If (residual_energy is medium) and (neighbors\# is many) and (centrality is close) then (chance is large)

10. If (residual_energy is high) and (neighbors\# is many) and (centrality is medium) then (chance is large)

From the fuzzy designed nets model, each node can get the fuzzy variable chance. The node with highest chance could be considered as a cluster head.

\section{Conclusion}

A novel algorithm in wireless sensor networks was designed. This system produce chance as an output, when three inputs are: Residual Energy, Number of Neighbors and Centrality. Considering various conditions in one system, the output would be more accurate and reliable. Moreover, fuzzy system increase the flexibility. Figure 5 illustrate the result of applying this system over 100 nodes with random distribution. 800 rounds is defined to get this output.

The network parameters are:

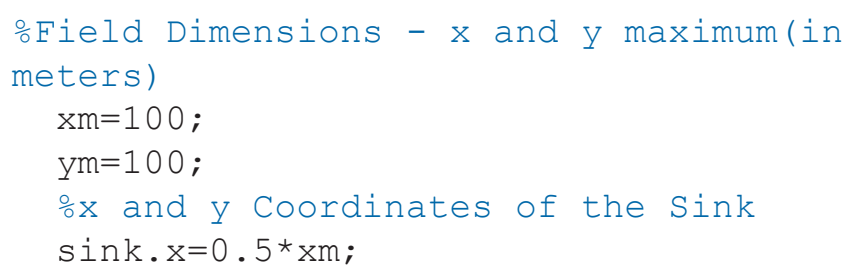


$\operatorname{sink} \cdot \mathrm{y}=0.5 * \mathrm{ym}$;

oNumber of Nodes in the field $\mathrm{n}=100$

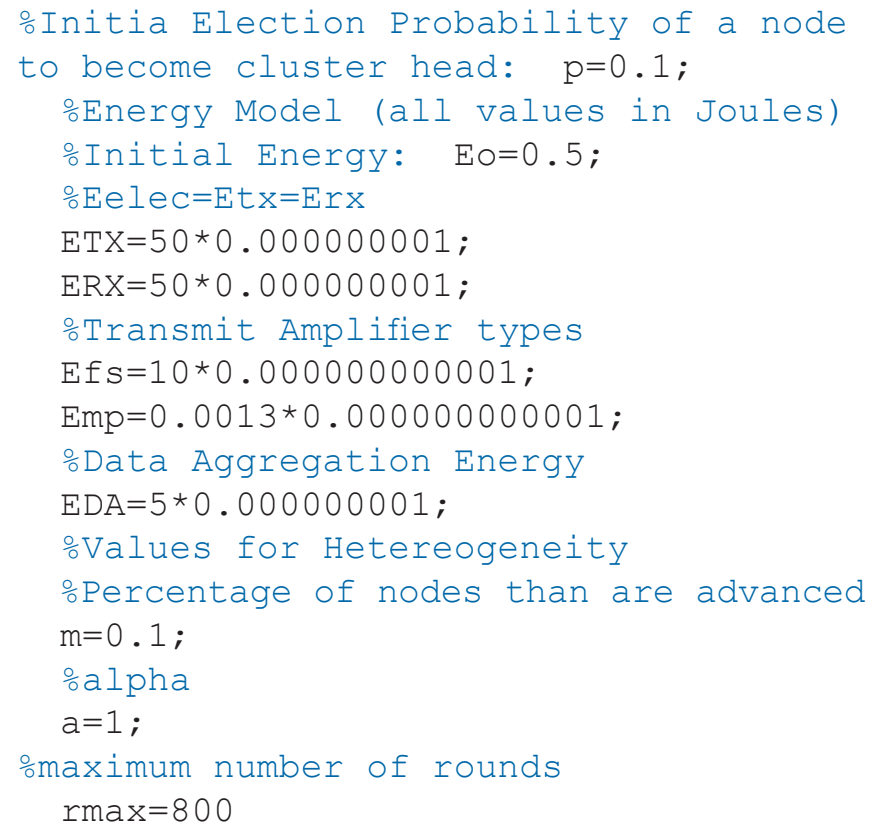

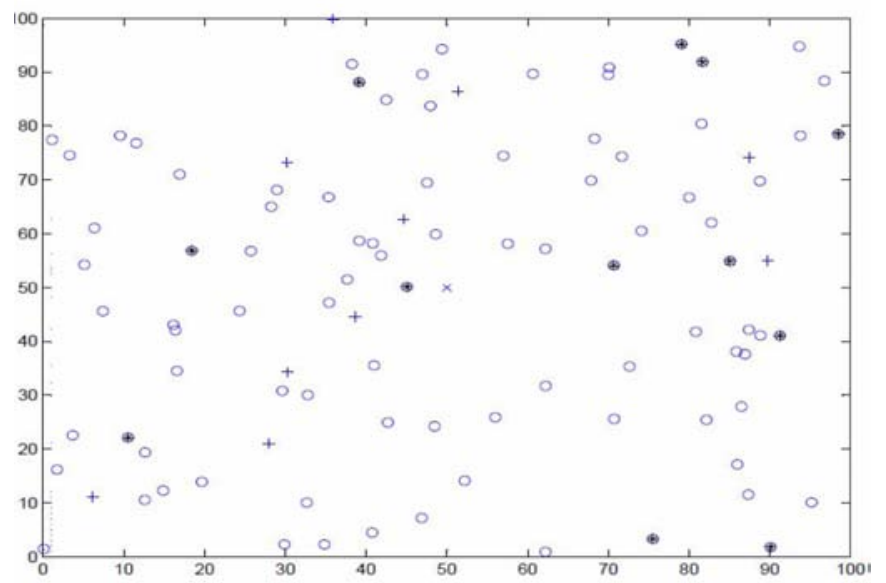

Figure 5. Obtained clusters over 100 randomly nodes.

Also alteration diagrams of some variables, include: distance, number of cluster head per round and probability, are shown in figure 6. Designed algorithm could be applied in different clustering method to increase accuracy and reliability.
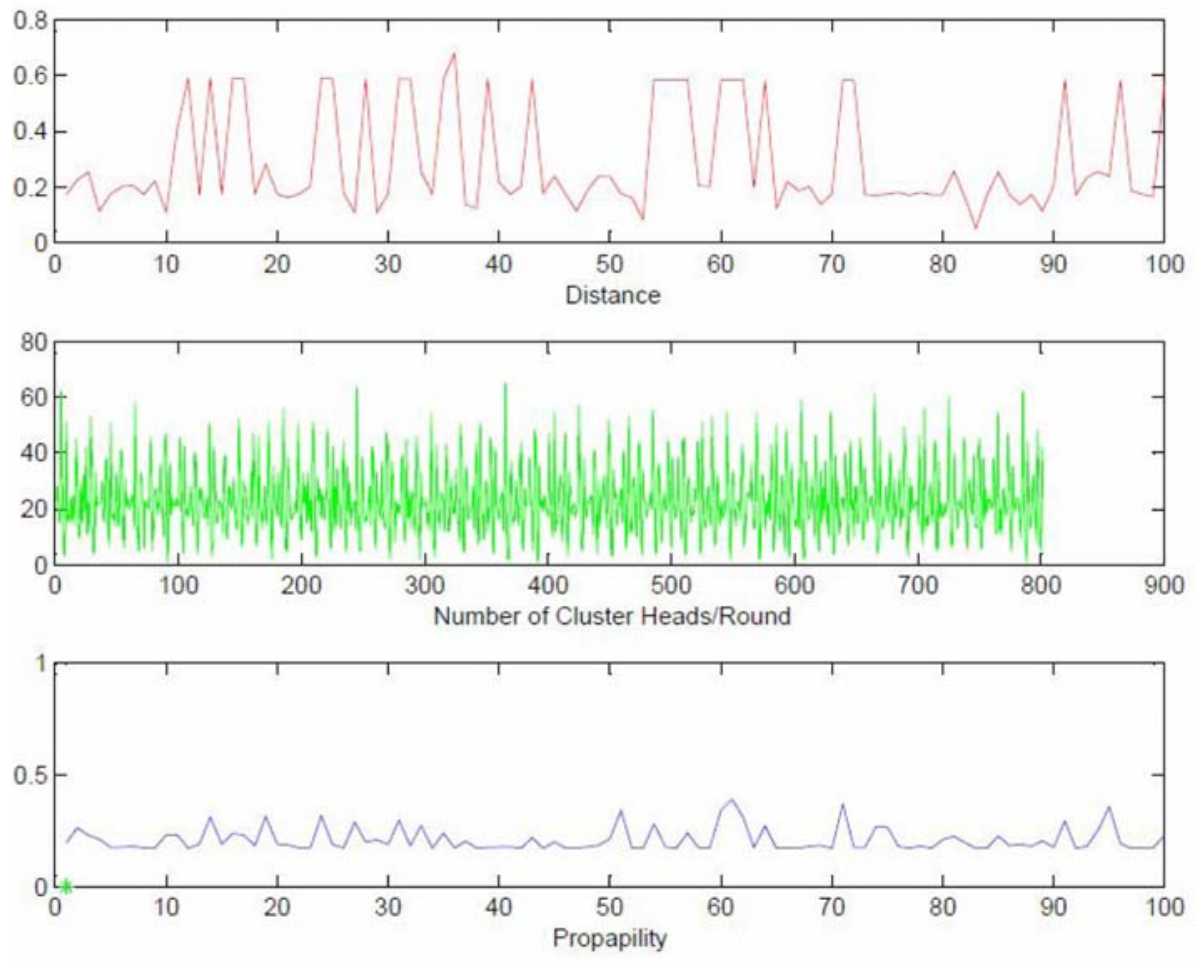

Figure 6. Diagrams of some parameters variations. 


\section{References}

1. Akyildiz L F, Su W et al. (2002). Wireless sensor networks: a survey, Computer Networks, vol 38(4), 393-422.

2. Yick J, Mukherjee B et al. (2008). Wireless sensor network survey, Computer Networks, 2008, vol 52(12), 2292-2330.

3. Abbasi A A, Younis M (2007). A survey on clustering algorithms for wireless sensor networks. Computer Communications, vol 30(14-15), 2826-2841.

4. Heinzelman W B, Chandrakasan A P et al. (2002). Application specific protocol architecture for wireless microsensor networks, IEEE Transactions on Wireless Communications, vol $1(4), 660-670$.
5. Lindsey S, and Raghavendra C S (2002). PEGASIS: Powerefficient gathering in sensor information systems, Proceedings of the IEEE Aerospace Conf. Montana: IEEE Aerospace and Electronic Systems Society, 1125-1130.

6. Younis O, and Fahmy S (2004). HEED: A hybrid, energy-efficient, distributed clustering approach for ad hoc sensor networks, IEEE Transactions on Mobile Computing, vol 3(4), 366-379.

7. Sivanandum S N, Sumathi S et al. (2007). Introduction to Fuzzy Logic using MATLAB, Springer-verlag, Berlin Heidelberg.

8. Fu F X, and Yu Z (2010). A reliable and efficient clustering algorithm for wireless sensor networks using Fuzzy Petri Nets, Wireless Communications Networking and Mobile Computing (WiCOM), 6th International Conference, 1-4. 